\title{
Comparison of acrosome reaction-inducing activities of human cumulus oophorus, follicular fluid and ionophore A23187 in human sperm populations of proven fertilizing ability in vitro
}

\author{
J. Tesařík* \\ Department of Histology and Embryology, Faculty of Medicine, J. E. Purkyně University, \\ tr. Obránců miru 10,662 43 Brno, Czechoslovakia
}

\begin{abstract}
Summary. Significant differences in the frequency of spontaneous and induced acrosome reactions (identified by electron microscopy) were detected between 5 individual sperm samples. Maximal stimulation of the acrosome reaction was achieved with ionophore A23187, no differences being detected between the two preincubation times used $(5$ and $15 \mathrm{~h})$. The cumulus oophorus and follicular fluid caused a similar increase in the proportion of acrosome-reacted spermatozoa after $5 \mathrm{~h}$ of preincubation. It is concluded that specific products of the cumulus and/or granulosa cells may contribute to the acrosome reaction-inducing activity of human follicular fluid.
\end{abstract}

\section{Introduction}

The acrosome reaction of mammalian spermatozoa, with its clearly defined morphological picture, is one of the physiologically relevant consequences of capacitation, a process which is much more difficult to trace. One of the most discussed aspects of mammalian sperm capacitation and the acrosome reaction is the question of participation of some products of ovulation in these events, since conflicting results have been reported (reviewed by Bavister, 1982; Bedford, 1983). Bovine follicular fluid has been shown to induce the acrosome reaction of hamster (Yanagimachi, 19,69) and mouse (Iwamatsu \& Chang, 1969) spermatozoa. Acrosome reaction-inducing activity has also been detected in the follicular fluid of a number of other mammalian species, including man (Mukerjee \& Lippes, 1972; Oliphant, Cabot \& Singhas, 1977). Although the bovine follicular fluid protein involved in the in-vitro induction of the hamster sperm acrosome reaction has been identified biochemically as serum albumin (Lui, Cornett \& Meizel, 1977), some studies suggest a possible role for more specific substances of follicular origin, probably produced by the cumylus cells, in the facilitation of the acrosome reaction in bovine (Lenz, Ax, Grimek \& First, 1982) and hamster (Bavister, 1982) spermatozoa. Furthermore, the binding of labelled glycoproteins secreted from cumulus cells to human spermatozoa has been shown to take place during incubation of the spermatozoa in conditions supporting capacitation in vitro (Tesaŕík, Kopečný \& Dvořa, 19ß4).

The present work attempts to evaluate the role of cumulus components in induction of the human sperm acrosome reaction. The effect of co-culture of spermatozoa with human cumuli oophori on the frequency of acrosome reactions is compared with the effect of addition of human follicular fluid and ionophore A23187 to the culture medium.

* Present address: Dr Jan Tesař́k, Station Centrale de Physiologie Animale, 78350 Jouy-en-Josas, France. 


\section{Materials and Methods}

Source and preparation of sperm suspensions. Semen samples were obtained from 5 healthy donors. All samples met the following standards: $>80 \times 10^{6}$ spermatozoa $/ \mathrm{ml} \mathrm{semen;}>70 \%$ motile spermatozoa; $>70 \%$ normal forms. After liquefaction, 8 subsamples of $0.2 \mathrm{ml}$ were taken from each sample. Spermatozoa were washed from seminal plasma by three cycles of centrifugation at $200 \mathrm{~g}$ for $10 \mathrm{~min}$ and resuspension in culture medium (see below), and actively moving spermatozoa were selected as previously described (Tesařik et al., 1984). All these centrifugations and incubations were carried out in plastic test tubes to avoid sticking of spermatozoa to glass surfaces, as has been described for spermatozoa from some mammalian species during washing procedures in the absence of albumin (Dott, Harrison \& Foster, 1979). Albumin contained in serum added to the culture medium (see below) may have further minimized the possibility of sperm selection by virtue of the washing procedures. The supernatants decanted after centrifugation were free of spermatozoa as assessed by occasional examinations under a dissecting microscope.

Experimental design and culture conditions. After the rinsing procedure, about $1 \times 10^{5}$ spermatozoa from each subsample were placed in a separate $50 \mu \mathrm{l}$ drop of Ham's F10 medium (Flow Laboratories, Rockville, MD, U.S.A.) supplemented with $15 \%(\mathrm{v} / \mathrm{v})$ heat-inactivated human fetal cord serum. The same sample of serum was used for all experiments. The medium was equilibrated with a gas phase of $90 \% \mathrm{~N}_{2}, 5 \% \mathrm{O}_{2}$, and $5 \% \mathrm{CO}_{2}(\mathrm{pH}$ of the equilibrated medium was 7.25-7.30). The cultures were carried out under paraffin oil in plastic Petri dishes so that 8 treatment drops with spermatozoa from one original sample were located in 2 dishes, each containing 4 drops. The dishes were then incubated in a closed culture box at $37^{\circ} \mathrm{C}$ in the above atmosphere. The following procedures were consistent for all sperm samples.

After $5 \mathrm{~h}$ of culture, one of the two dishes containing material from 1 sample was taken out of the culture box, forming the basis for Treatments 1-4 (Table 1). A fully expanded cumulus mass obtained from a human preovulatory follicle (as described by Tesařik et al., 1984) was rinsed three times in the culture medium and added to one drop (Treatment 2, Table 1) while $20 \mu$ l human follicular fluid (from the same follicle as the cumulus) and ionophore A23187 (Sigma, St Louis, MO, U.S.A.; final concentration of $15 \mu \mathrm{M}$ ) were introduced into two other drops (Treatments 3 and 4, Table 1). Basal culture medium $(20 \mu \mathrm{l})$ was added to the remaining drop which served as a control (Treatment 1, Table 1). After incubation for an additional $1 \mathrm{~h}$, the contents of the 4 treatment drops were transferred into plastic test tubes, diluted with $1 \mathrm{ml}$ fresh culture medium, and centrifuged at $200 \mathrm{~g}$ for $10 \mathrm{~min}$. Treatment drops 5-8, contained in the other Petri dish, were treated in the same way after $15 \mathrm{~h}$ of preculture (Table 1 ).

To test the effects of the different treatments on sperm motility, $5 \mu l$ were taken from each drop at the end of the preculture period and again after the incubation with the component tested. Motility was evaluated under dark-ground illumination using $1 \mathrm{sec}$ timed-exposure photomicrography (Overstreet, Katz, Hanson \& Fonseca, 1979). Tracks of 100 spermatozoa were analysed, and percentages of motile spermatozoa were recorded. Progressively and non-progressively moving spermatozoa were considered motile.

Aliquants of all the 5 original sperm samples investigated were used for in-vitro insemination of human oocytes obtained during diagnostic laparoscopy (Pilka, Trávník \& Tesařik, 1983). These experiments were done with the informed consent of the patients and the approval of the hospital ethical committee. All the eggs were successfully fertilized and at least one egg per sperm sample cleaved to the 4-cell stage.

Electron microscopy. The pellets of spermatozoa obtained as indicated above were fixed with $3 \%$ glutaraldehyde in $0.1 \mathrm{M}$-cacodylate buffer, followed by $1 \%$ osmium tetroxide in the same buffer, dehydrated and embedded in Durcupan ACM. Thin sections cut at 7-10 randomly chosen section planes were prepared from each pellet. They were viewed in a Tesla BS 500 electron microscope. 
Assessment of acrosome reaction frequency. The head profiles of spermatozoa in each pellet were counted. Only spermatozoa with heads of normal shape and lacking any obvious morphological abnormalities were included. Each of the 300 spermatozoa counted in each pellet was categorized as acrosome-reacted or non-acrosome-reacted. The criteria for an acrosome-reacted spermatozoon were the selective disintegration or total absence of the plasma and outer acrosomal membranes in the anterior region of the sperm head (vesicles originating through their fusion might or might not be present in the vicinity of the acrosome-reacted sperm head), and the integrity of the plasma membrane covering the equatorial segment. The fusion of the plasma membrane covering the equatorial segment with the inner acrosomal membrane at the boundary between the anterior acrosomal and equatorial regions was another criterion of a normal acrosome reaction. Only sperm heads for which all these features could be evaluated were counted. The category of acrosomereacted spermatozoa therefore included spermatozoa that had undergone an acrosome reaction and those just undergoing this process. Spermatozoa with a disintegrated plasma membrane over the entire head were considered to be dead cells and were excluded from the study, whereas those retaining the intact plasma and outer acrosomal membranes were classified as non-acrosomereacted even if some degree of acrosomal swelling could be identified in some of them.

Statistical analysis of data. The percentages of acrosome-reacted spermatozoa were calculated for each sample and each treatment regimen. The proportional data were transformed into angles by the arc-sin transformation. Analysis of variance was performed for two-way classification within individual treatments (lines, Table 1). The $\chi^{2}$ test was used to determine the statistical significance of differences between treatments for each sperm sample (columns, Table 1). Both analyses were performed using a computing programme.

\section{Results}

The addition to the culture medium of any of the factors tested after $5 \mathrm{~h}$ of sperm preincubation led to a significant $(P<0.01)$ increase in the proportion of acrosome-reacted spermatozoa in comparison with controls (Table 1). The most efficient enhancement of the acrosome reaction was achieved using the ionophore A23187. The incidence of the acrosome reaction was similar when the cumulus oophorus or follicular fluid were added to the culture medium $(P>0.05)$.

When spermatozoa were precultured in the capacitating medium for $15 \mathrm{~h}$, the enhancement of the acrosome-reaction rate in suspensions subsequently exposed to the cumulus oophorus or follicular fluid was significant only in 3 of the 5 samples tested if compared with the controls (Table 1). The incidence of acrosome-reacted spermatozoa incubated with the cumulus oophorus and

Table 1. Effects of cumulus oophorus, follicular fluid (FF), and A23187 on the percentage of acrosomereacted human spermatozoa

\begin{tabular}{|c|c|c|c|c|c|c|c|}
\hline \multirow[b]{2}{*}{ Treatment } & \multirow{2}{*}{$\begin{array}{l}\text { Sperm } \\
\text { preculture } \\
\text { time (h) }\end{array}$} & \multirow{2}{*}{$\begin{array}{l}\text { Component } \\
\text { added }\end{array}$} & \multicolumn{5}{|c|}{$\%$ of acrosome-reacted spermatozoa } \\
\hline & & & Sample 1 & Sample 2 & Sample 3 & Sample 4 & Sample 5 \\
\hline 1 & 5 & None & $5 \cdot 0^{3}$ & $8.7^{\mathrm{a}}$ & $11 \cdot 3^{\mathrm{a}}$ & $18 \cdot 3^{\mathrm{a}}$ & $25 \cdot 7^{\mathrm{a}}$ \\
\hline 2 & 5 & Cumulus & $10^{0} 7^{\mathrm{b}}$ & $17 \cdot 7^{\mathrm{b}, \mathrm{c}}$ & $34 \cdot 0^{b}$ & $29 \cdot 7^{b}$ & $38.0^{\mathrm{b}}$ \\
\hline 3 & 5 & FF & $14 \cdot 3^{b}$ & $20 \cdot 0^{\mathrm{b}}$ & $36 \cdot 7^{\mathrm{b}, \mathrm{c}}$ & $32 \cdot 3^{\mathrm{b}, \mathrm{c}}$ & $34 \cdot 7^{b}$ \\
\hline 4 & 5 & A23187 & $61 \cdot 3^{c}$ & $44 \cdot 3^{d}$ & $59 \cdot 3^{\mathrm{d}}$ & $54 \cdot 7^{d}$ & $66 \cdot 7^{c, d}$ \\
\hline 5 & 15 & None & $6 \cdot 0^{a}$ & $12 \cdot 7^{\mathrm{a}, \mathrm{c}}$ & $22.7^{\mathrm{e}}$ & $36 \cdot 7^{b . c}$ & $58 \cdot 0^{\mathrm{e}}$ \\
\hline 6 & 15 & Cumulus & $40 \cdot 3^{d}$ & $36 \cdot 0^{e}$ & $38 \cdot 7^{b, c}$ & $37 \cdot 3 c$ & $62 \cdot 0^{\mathrm{c}, \mathrm{d}, \mathrm{e}}$ \\
\hline 7 & 15 & FF & $36 \cdot 3^{\mathrm{d}}$ & $32 \cdot 3^{\mathrm{e}}$ & $44 \cdot 3^{c}$ & $38 \cdot 3^{\mathrm{c}}$ & $59 \cdot 3^{\text {c.e }}$ \\
\hline 8 & 15 & A23187 & $66 \cdot 3^{\mathrm{c}}$ & $47 \cdot 3^{\mathrm{d}}$ & $61 \cdot 0^{\mathrm{d}}$ & $60 \cdot 3^{\mathrm{d}}$ & $68 \cdot 0^{d}$ \\
\hline
\end{tabular}

a,b,c,d,e Values with at least one common superscript symbol within each column are not significantly different from each other $(P>0.05)$. 
Table 2. Effects of different treatment regimens on human sperm motility

\begin{tabular}{cccccc}
\hline & \multicolumn{5}{c}{$\begin{array}{c}\text { \% of motile spermatozoa before and after } \\
\text { incubation with the component tested } \dagger\end{array}$} \\
\cline { 2 - 6 } Treatment* & Sample 1 & Sample 2 & Sample 3 & Sample 4 & Sample 5 \\
\hline 1 & $96 / 95$ & $94 / 93$ & $97 / 98$ & $94 / 90$ & $97 / 92$ \\
2 & $98 / 96$ & $93 / 90$ & $98 / 95$ & $93 / 88$ & $99 / 90$ \\
3 & $98 / 97$ & $96 / 91$ & $97 / 94$ & $96 / 91$ & $99 / 95$ \\
4 & $95 / 10$ & $96 / 12$ & $93 / 9$ & $93 / 5$ & $95 / 6$ \\
5 & $80 / 78$ & $79 / 75$ & $78 / 75$ & $76 / 73$ & $54 / 50$ \\
6 & $82 / 76$ & $81 / 75$ & $74 / 69$ & $72 / 64$ & $62 / 57$ \\
7 & $84 / 77$ & $86 / 78$ & $76 / 68$ & $75 / 68$ & $55 / 48$ \\
8 & $79 / 5$ & $80 / 8$ & $78 / 6$ & $74 / 4$ & $59 / 2$ \\
\hline
\end{tabular}

* See Table 1 for explanation of treatment regimens.

$\dagger$ Motile spermatozoa after preculture but before the experimental incubation/ motile spermatozoa after the experimental incubation.

follicular fluid after $15 \mathrm{~h}$ of preincubation (Treatments 6 and 7, Table 1) was elevated significantly in comparison with the corresponding treatment groups after $5 \mathrm{~h}$ of sperm preincubation (Theatments 2 and 3, Table 1) in 3 of the 5 samples used. In one sample (Sample 4, Table 1) this difference was significant only for the cumulus oophorus as the component tested. By contrast, the different time of sperm preincubation did not produce any significant difference in the frequency of acrosome reactions when the spermatozoa were subsequently exposed to A23187.

In 3 of the 5 sperm samples tested the frequency of spontaneous acrosome reactions after $15 \mathrm{~h}$ of sperm culture was significantly higher than that after $5 \mathrm{~h}$ of culture (Table 1 ), reaching as much as $58 \%$ of the total sperm population. The variation of acrosome-reaction rates between samples was significant in all treatment groups, indicating an expressed intrinsic variability of the sperm populations.

Motility was higher than $90 \%$ in all sperm samples after $5 \mathrm{~h}$ of preculture and higher than $50 \%$ in all the samples after $15 \mathrm{~h}$ of preculture (Table 2). The differences in the reduction of sperm motility after incubation with the component tested between the treatment regimens did not exceed $5 \%$ except in the incubations with A23187 which reduced the sperm motility dramatically (Table 2).

\section{Discussion}

The results of this study demonstrate a significant enhancement of human sperm acrosome reaction rates in the presence of human cumulus oophorus and follicular fluid after $5 \mathrm{~h}$ of preincubation of thè spermatozoa in medium supporting capacitation in vitro. The similar percentages of acrosomereacted spermatozoa in the experiments involving the addition of the cumulus oophorus and follicular fluid suggest that the acrosome reaction may be facilitated by an identical substance coftained in both entities and that serum albumin is not this active component. Indeed, all media used in this study contained human fetal cord serum whose albumin fraction reached the concentration commonly used to supplement media for in-vitro fertilization. The assumed actosome reaction-inducing substance may be related to the cumulus cell-secreted glycoproteins thă bind to human spermatozoa during capacitation in vitro (Tesařik et al., 1984).

The maximal stimulation of the acrosome reaction with the divalent cation ionophore A23187 whlich has been shown to increase the intracellular levels of calcium in treated mammalian sperrmatozoa (Babcock, First \& Lardy, 1976; Singh, Babcock \& Lardy, 1978) confirms a key role of calcium in the human sperm acrosome reaction. Moreover, the proportional data indicate that the 
acrosome reaction can be achieved in this artificial way even in those spermatozoa that would not do so either spontaneously or in the presence of the two "natural' acrosome reaction-facilitating agents within the given time period. This finding is consistent with the observations that capacitation of eutherian spermatozoa becomes unnecessary when calcium is driven in by this or other means (Yanagimachi, 1975; Talbot, Summers, Hylander, Keough \& Franklin, 1976; Green, 1978).

By contrast to A23187 which by-passes capacitation, the other two agents tested do not seem to induce the acrosome reaction synchronously but gradually. Because the putative receptors for the zona pellucida are supposed to be located on the plasma membrane of human sperm heads (Dvorák, Tesařík \& Kopečný, 1984) and motility of mammalian spermatozoa probably wanes within about $2 \mathrm{~h}$ after the acrosome reaction (Bedford, 1983), the flexibility in the timing of capacitation may serve a physiological basis for an appropriate staggering of acrosome-reaction time among the sperm population, resulting in a maximal prolongation of the interval for which potentially fertilizing spermatozoa colonize the oviducal ampulla. The induction of the acrosome reaction by the two products of ovulation, as demonstrated in this study, may then support the fertilizing ability of spermatozoa at the site and time of ovulation under conditions in vivo.

A shortcoming of this study may be that no functional tests of membrane integrity based on dye exclusion were included. However, the integrity of the plasma membrane covering the equatorial segment and fusion of this plasma membrane with the intact inner acrosomal membrane at the boundary between the anterior acrosomal and equatorial regions, as revealed by electron microscopy, may represent a morphological counterpart of the ability of spermatozoa to exclude vital dyes, although this correlation has not been sufficiently proved. This morphological picture is usually attributed to a 'true' acrosome reaction while 'false' acrosome reactions as a result of damage to spermatozoa often involve the loss of individual head membranes as separate entities (Bedford \& Cooper, 1978). Furthermore, even if post-mortem acrosome reactions were included in the counts of acrosome-reacted spermatozoa, these errors cannot have significantly influenced the results of the experiments with the cumulus oophorus and follicular fluid because the differences between sperm motility before and after incubation were relatively small and similar in all these experiments (see Table 2). On the other hand, sperm motility was significantly reduced after the incubations with A23187, but this is a commonly known effect of this ionophore on mammalian spermatozoa.

Another important feature of human ejaculated sperm populations revealed by the present study is the expressed variability of both spontaneous and induced acrosome reaction frequencies between individual samples. Because successful in-vitro fertilization was achieved with samples of all the sperm populations used, the differences in the acrosome-reaction pattern between the samples may reflect the variation between donors in the time required for semen samples to express maximum fertilizing ability when assessed using a zona-free hamster egg assay (Perreault \& Rogers, 1982). The biochemical peculiarities underlying the differences between donors remain to be clarified.

Ionophore A23187 was kindly supplied by Dr J. E. Fléchon from the Central Station of Animal Physiology, INRA, Jouy-en-Josas, France, who has also critically reviewed the manuscript. I thank Dr J. Holčík and Dr K. Drdla for performing the statistical analyses, and Mrs E. Golová for technical assistance.

\section{References}

Babcock, D.F., First, N.L. \& Lardy, H.A. (1976) Action of ionophore A23187 at the cellular level. Separation of effects at the plasma and mitochondrial membranes. J. biol. Chem. 251, 3881-3886.
Bavister, B.D. (1982) Evidence for a role of postovulatory cumulus components in supporting fertilizing ability of hamster spermatozoa. J. Androl. 3, 365-372.

Bedford, J.M. (1983) Significance of the need for sperm Downloaded from Bioscientifica.com at 04/26/2023 07:29:30AM 
capacitation before fertilization in eutherian mammals. Biol. Reprod. 28, 108-120.

Bedford, J.M. \& Cooper, G.W. (1978) Membrane fusion events in the fertilization of vertebrate eggs. In Membrane Fusion, pp. 65-125. Eds G. Poste \& G. L. Nicolson. Elsevier/North-Holland Biomedical Press, Amsterdam.

Dott, H.M., Harrison, R.A.P. \& Foster, G.C.A. (1979) The maintenance of motility and the surface properties of epididymal spermatozoa from bull, rabbit and ram in homologous seminal and epididymal plasma. J. Reprod. Fert. 55, 113-124.

Dvởák, M., Tesaňk, J. \& Kopečný, V. (1984) Ultrastructure of human fertilization. In Ultrastructure of Reproduction, pp. 176-189. Eds J. Van Blerkom \& P. M. Motta. Martinus Nijhoff, Boston.

Green, D.P.L. (1978) The induction of the acrosome reaction in guinea-pig sperm by the divalent metal cation ionophore A23187. J. Cell Sci. 32, 137-151.

Iwamatsu, T. \& Chang, M.C. (1969) In vitro fertilization of mouse eggs in the presence of bovine follicular fluid. Nature, Lond. 224, 919-920.

Lenz, R.W., Ax, R.L., Grimek, H.J. \& First, N.L. (1982) Proteoglycan from bovine follicular fluid stimulates an acrosome reaction in bovine spermatozoa. Biochem. Biophys. Res. Commun. 106, 1092-1098.

Lui, C.W., Cornett, L.E. \& Meizel, S. (1977) Identification of the bovine follicular fluid protein involved in the in vitro induction of the hamster sperm acrosome reaction. Biol. Reprod. 17, 34-41.

Mukerjee, A.B. \& Lippes, J. (1972) Effect of human follicular and tubal fluids on human, mouse, and rat spermatozoa in vitro. Can. J. Genet. Cytol. 14, 167174.
Oliphant, G., Cabot, C.L. \& Singhas, C.A. (1977) Nature of the rabbit acrosome reaction-inducing activity of follicular fluid. J. Reprod. Fert. 50, 245-250.

Overstreet, J.W., Katz, D.F., Hanson, F.W. \& Fonseca, J.R. (1979) A simple inexpensive method for objective assessment of human sperm movement characteristics. Fert. Steril. 31, 162-172.

Perreault, S.D. \& Rogers, B.J. (1982) Capacitation pattern of human spermatozoa. Fert. Steril. 38, 258260.

Pilka, L., Trávník, P. \& Tesař́k, J. (1983) Diagnose der grundlegenden Sterilitätsursache des Ehepaares mittels einer laparoskopischen Untersuchung. Zentbl. Gynäkol. 105, 487-492.

Singh, J.P., Babcock, D.F. \& Lardy, H.A. (1978) Increased calcium-ion influx is a component of capacitation of spermatozoa. Biochem. J. 172, 549556.

Talbot, P., Summers, R.G., Hylander, B.L., Keough, E.M. \& Franklin, L.E. (1976) The role of calcium in the acrosome reaction: an analysis using ionophore A23187. J. exp. Zool. 198, 383-392.

Tesařik, J., Kopečný, V. \& Dvořăk, M. (1984) Selective binding of human cumulus cell-secreted glycoproteins to human spermatozoa during capacitation in vitro. Fert. Steril. 41, 919-925.

Yanagimachi, R. (1969) In vitro acrosome reaction and capacitation of golden hamster spermatozoa with bovine follicular fluid and its fractions. J. Reprod. Fert. 18, 275-286.

Yanagimachi, R. (1975) Acceleration of the acrosome reaction and activation of guinea-pig spermatozoa by detergents and other reagents. Biol. Reprod. 13, 519 526.

Received 24 July 1984 